Journal of Nonlinear Mathematical Physics

\title{
On the Propagation of Binary Signals in a Two-Dimensional Nonlinear Lattice with Nearest-Neighbor Interactions
}

Javier Ruiz-Ramírez, Jorge Eduardo Macías-Díaz

To cite this article: Javier Ruiz-Ramírez, Jorge Eduardo Macías-Díaz (2010) On the Propagation of Binary Signals in a Two-Dimensional Nonlinear Lattice with NearestNeighbor Interactions, Journal of Nonlinear Mathematical Physics 17:2, 127-136, DOI: https://doi.org/10.1142/S1402925110000659

To link to this article: https://doi.org/10.1142/S1402925110000659

Published online: 04 January 2021 


\title{
ON THE PROPAGATION OF BINARY SIGNALS IN A TWO-DIMENSIONAL NONLINEAR LATTICE WITH NEAREST-NEIGHBOR INTERACTIONS
}

\author{
JAVIER RUIZ-RAMÍREZ \\ Departamento de Bioquímica \\ Universidad Autónoma de Aguascalientes \\ Avenida Universidad 940, Ciudad Universitaria \\ Aguascalientes 20100, Mexico \\ jruiz@correo.uaa.mx \\ JORGE EDUARDO MACÍAS-DÍAZ \\ Departamento de Matemáticas y Física \\ Universidad Autónoma de Aguascalientes \\ Avenida Universidad 940, Ciudad Universitaria \\ Aguascalientes 20100, Mexico \\ jemacias@correo.uaa.mx
}

Received 12 August 2009

Accepted 28 October 2009

\begin{abstract}
In this work, we use a computational technique with multiple properties of consistency in order to approximate solutions of a bounded $\beta$-Fermi-Pasta-Ulam lattice in two space dimensions subject to harmonic driving in two adjacent boundaries. The processes of nonlinear supratransmission and infratransmission are employed in order to propagate binary signals in the medium by means of a suitable modulation of the driving amplitude.
\end{abstract}

Keywords: Two-dimensional lattice; Fermi-Pasta-Ulam system; finite difference scheme; harmonic boundary perturbation; wave propagation; binary signals.

\section{Introduction}

The process of supratransmission consists in the sudden increase in the amplitude of wave signals transmitted into a nonlinear medium perturbed by a harmonic source, irradiating at a frequency in the forbidden band gap. The process was discovered in discrete chains of harmonic oscillators coupled by springs satisfying Hooke's law [9], where the governing law consists of a system of coupled sine-Gordon equations with Dirichlet boundary data. Later on, it was shown that the process is likewise present in superconductivity problems, particularly, in discrete Josephson-junction arrays coupled through superconducting wires and perturbed sinusoidally by an external source [5], where the regime is again a system of coupled sine-Gordon equations, this time with a Neumann boundary condition. 
Nonlinear supratransmission has been investigated in other $(1+1)$-dimensional physical models. For instance, it has been shown that supratransmission is present in models consisting of discrete Klein-Gordon equations [21, 9], double sine-Gordon equations [10], Bragg media in the nonlinear Kerr regime [23], and even in continuous problems governed by sine-Gordon equations subject to Dirichlet [26] or Neumann boundary data [5]. Moreover, this nonlinear phenomenon is present in several $(2+1)$-dimensional systems $[17,16,15]$, and several applications have been proposed for one-dimensional scenarios, including applications to the transmission of binary signals in nonlinear media [18-20], as well as for two-dimensional [16] and three-dimensional cases [15].

Supratransmission in $(1+1)$-dimensional chains of anharmonic oscillators has also been investigated in the literature [27]. The existence of this process together with regions of bistability where conducting and insulating regimes coexist has been approximated analytically and numerically, as it was previously done for the continuous sine-Gordon equation [26]. More precisely, it has been established that the classical $\beta$-Fermi-Pasta-Ulam chains studied in [7] (a model that has been studied extensively in more general settings $[4,8]$ ) are capable of transmitting energy in the form of localized nonlinear modes [22] when the boundary is perturbed harmonically at a frequency in the forbidden band-gap. Moreover, the existence of a bistable regime within the forbidden band-gap of the model was approximated both analytically and computationally.

In this work, we present computational evidence of the possibility to transmit binary information in a generalization of the $(2+1)$-dimensional version of the problem studied in [27], using nonlinear bistability. There exist many possible generalizations of the original model studied by Fermi, Pasta and Ulam in 1955, including generalizations to square [13] and hexagonal [14] lattices; however, in this paper, we study the simplest generalization of the $\beta$-Fermi-Pasta-Ulam chain, namely, the generalization studied in [29]. Our results are based on a numerical method with consistency properties in the domains of both solution and energy, and they establish the feasibility to transmit binary bits in the medium under study by employing the phenomena of nonlinear supratransmission and infratransmission.

\section{Mathematical Model}

Let $N$ be a positive integer, and for every $m, n=0,1, \ldots, N$, let $u_{m, n}(t)$ be a function of time $t \geq 0$. Let $\gamma$ be a nonnegative constant, and let $\beta$ and $c$ be positive real numbers. In this paper we consider the following initial-value problem with boundary data, for $m, n=$ $1,2, \ldots, N-1$ :

$$
\begin{aligned}
& \frac{d^{2} u_{m, n}}{d t^{2}}=-\gamma \frac{d u_{m, n}}{d t}+c^{2}\left(\delta_{x}^{2}+\delta_{y}^{2}\right) u_{m, n}+\beta\left[\left(\delta_{x} u_{m, n}\right)^{3}\right. \\
& \left.-\left(\delta_{x} u_{m, n-1}\right)^{3}+\left(\delta_{y} u_{m, n}\right)^{3}-\left(\delta_{y} u_{m, n-1}\right)^{3}\right], \\
& \text { s.t. } \begin{cases}\frac{d u_{m, n}(0)}{d t}=u_{m, n}(0)=0, & \\
u_{m, 0}(t)=u_{0, n}(t)=\phi(t), & t \geq 0, \\
\delta_{x} u_{N-1, n}(t)=\delta_{y} u_{m, N-1}(t)=0, & t \geq 0 .\end{cases}
\end{aligned}
$$

Clearly, the constant $\gamma$ assumes the role of damping, while $c$ and $\beta$ are called the coefficients of coupling and nonlinearity, respectively. For practical reasons, the function $\phi$ 
may be thought of as a sinusoidal perturbation at the boundary, namely,

$$
\phi(t)=A \sin (\Omega t)
$$

where $\Omega>2$, following [27]. Meanwhile, the difference operators employed in (2.1) are given by

$$
\begin{aligned}
& \delta_{x} u_{m, n}=u_{m+1, n}-u_{m, n}, \\
& \delta_{y} u_{m, n}=u_{m, n+1}-u_{m, n}, \\
& \delta_{x}^{2} u_{m, n}=u_{m+1, n}-2 u_{m, n}+u_{m-1, n}, \\
& \delta_{y}^{2} u_{m, n}=u_{m, n+1}-2 u_{m, n}+u_{m, n-1} .
\end{aligned}
$$

These operators are assumed to be linear, and their compositions are defined in the usual way.

Obviously, (2.1) is a generalization to the $(2+1)$-dimensional scenario, of the $\beta$-Fermi-Pasta-Ulam model studied in [7], which is a model that possesses many applications $[12,30,24,31,2,11,25]$, and which has been extensively studied in the literature of nonlinear science $[28,6,1,3]$. It is the simplest generalization to two space dimensions [29]. It describes a discrete, two-dimensional system of particles, each of them governed by the corresponding ordinary differential equation of (2.1), initially at rest, and where the boundary is harmonically perturbed with a frequency $\Omega$ and an amplitude $A$.

It is not difficult to check that the Hamiltonian of system (2.1) is given by

$$
H_{m, n}=\frac{1}{2}\left(\frac{d u_{m, n}}{d t}\right)^{2}+\frac{c^{2}}{2}\left[\left(\delta_{x} u_{m, n}\right)^{2}+\left(\delta_{y} u_{m, n}\right)^{2}\right]+\frac{\beta}{4}\left[\left(\delta_{x} u_{m, n}\right)^{4}+\left(\delta_{y} u_{m, n}\right)^{4}\right]
$$

for every $m, n=1,2, \ldots, N-1$. After including the potential functions between the couplings in the boundaries of the system, it can be checked that the total energy of the medium at any time is given by

$$
\begin{aligned}
E= & \sum_{m=1}^{N-1} \sum_{n=1}^{N-1} H_{m, n}+\frac{c^{2}}{2}\left[\sum_{m=1}^{N-1}\left(\delta_{y} u_{m, 0}\right)^{2}+\sum_{n=1}^{N-1}\left(\delta_{x} u_{0, n}\right)^{2}\right] \\
& +\frac{\beta}{4}\left[\sum_{m=1}^{N-1}\left(\delta_{y} u_{m, 0}\right)^{4}+\sum_{n=1}^{N-1}\left(\delta_{x} u_{0, n}\right)^{4}\right] .
\end{aligned}
$$

Moreover, it turns out that the rate of change of the total energy of the system with respect to time is given by the expression

$$
\begin{aligned}
\frac{d E}{d t}= & -\gamma \sum_{m=1}^{N-1} \sum_{n=1}^{N-1}\left(\frac{d u_{m, n}}{d t}\right)^{2}-\sum_{m=1}^{N-1}\left(u_{m, 1}-u_{m, 0}\right)\left[c^{2}+\beta\left(u_{m, 1}-u_{m, 0}\right)^{2}\right] \frac{d u_{m, 0}}{d t} \\
& -\sum_{n=1}^{N-1}\left(u_{1, n}-u_{0, n}\right)\left[c^{2}+\beta\left(u_{1, n}-u_{0, n}\right)^{2}\right] \frac{d u_{0, n}}{d t} .
\end{aligned}
$$

\section{Numerical Method}

Let $T$ be a positive real number. In order to approximate solutions to $(2.1)$ on the time interval $[0, T]$, we fix a regular partition $0=t_{0}<t_{1}<\cdots<t_{M}=T$ with norm equal 
to $\Delta t=T / M$, and let $u_{m, n}^{k}$ represent the approximation provided by our method to the actual value of the solution $u_{m, n}$ of problem (2.1) at time $t_{k}$, for every $k=0,1, \ldots, M$. We introduce here the linear operators

$$
\begin{aligned}
& \delta_{t} u_{m, n}^{k}=u_{m, n}^{k+1}-u_{m, n}^{k}, \\
& \delta_{t}^{1} u_{m, n}^{k}=u_{m, n}^{k+1}-u_{m, n}^{k-1}, \\
& \delta_{t}^{2} u_{m, n}^{k}=u_{m, n}^{k+1}-2 u_{m, n}^{k}+u_{m, n}^{k-1},
\end{aligned}
$$

for every $m, n=0,1, \ldots, N$ and $k=1,2, \ldots, N-1$. Moreover, we introduce the nonstandard operators

$$
\begin{aligned}
\sigma_{x} u_{m, n} & =\sum_{j=0}^{3}\left(\delta_{x} u_{m, n}^{k+1}\right)^{j}\left(\delta_{x} u_{m, n}^{k}\right)^{3-j}, \\
\sigma_{y} u_{m, n} & =\sum_{j=0}^{3}\left(\delta_{y} u_{m, n}^{k+1}\right)^{j}\left(\delta_{y} u_{m, n}^{k}\right)^{3-j} .
\end{aligned}
$$

The finite-difference scheme proposed in this paper to approximate solutions of initialvalue problem with boundary data $(2.1)$ is given by the following system of difference equations, for $m, n=1,2, \ldots, N-1$ and $k=2,3, \ldots, M-2$ :

$$
\begin{gathered}
\frac{\delta_{t}^{2} u_{m, n}^{k+1}+\delta_{t}^{2} u_{m, n}^{k}}{2 \Delta t}=-\gamma \frac{\delta_{t}^{1} u_{m, n}^{k}}{2 \Delta t}+c^{2} \frac{\delta_{x}^{2} u_{m, n}^{k+1}+\delta_{x}^{2} u_{m, n}^{k}}{2}+c^{2} \frac{\delta_{y}^{2} u_{m, n}^{k+1}+\delta_{y}^{2} u_{m, n}^{k}}{2} \\
\quad+\beta \frac{\sigma_{x} u_{m, n}^{k}+\sigma_{x} u_{m-1, n}^{k}}{4}+\beta \frac{\sigma_{y} u_{m, n}^{k}+\sigma_{y} u_{m, n-1}^{k}}{4}, \\
\text { s.t. } \begin{cases}u_{m, n}^{1}=u_{m, n}^{0}=0, & k=0,1, \ldots, M, \\
u_{m, 0}^{k}=u_{0, n}^{k}=\phi\left(t_{k}\right), & k=0,1, \ldots, M .\end{cases}
\end{gathered}
$$

It is readily checked that (3.3) provides consistent approximations of the solutions of (2.1). Moreover, the local energy density in the node $(m, n)$ of our problem is also consistently approximated using the discrete expression

$$
\begin{aligned}
H_{m, n}^{k}= & \frac{\left(\delta_{t} u_{m, n}^{k}\right)\left(\delta_{t} u_{m, n}^{k-1}\right)}{2(\Delta t)^{2}}+c^{2} \frac{\left(\delta_{x} u_{m, n}^{k}\right)^{2}+\left(\delta_{x} u_{m-1, n}^{k}\right)^{2}}{4}+c^{2} \frac{\left(\delta_{y} u_{m, n}^{k}\right)^{2}+\left(\delta_{y} u_{m, n-1}^{k}\right)^{2}}{4} \\
& +\beta \frac{\left(\delta_{x} u_{m, n}^{k}\right)^{4}+\left(\delta_{x} u_{m-1, n}^{k}\right)^{4}}{8}+\beta \frac{\left(\delta_{y} u_{m, n}^{k}\right)^{4}+\left(\delta_{y} u_{m, n-1}^{k}\right)^{4}}{8}
\end{aligned}
$$

In turn, the total energy $E^{k}$ of the system at time $t_{k}$ is calculated by adding all the discrete Hamiltonians to the potentials of the couplings to the nodes of the boundaries. In other words, the discrete energy associated to the finite-difference scheme (3.3) is given by

$$
\begin{aligned}
E^{k}= & \sum_{m=1}^{N-1} \sum_{n=1}^{N-1} H_{m, n}^{k}+\frac{c^{2}}{4}\left[\sum_{m=1}^{N-1}\left(\delta_{y} u_{m, 0}^{k}\right)^{2}+\sum_{n=1}^{N-1}\left(\delta_{x} u_{0, n}^{k}\right)^{2}\right] \\
& +\frac{\beta}{8}\left[\sum_{m=1}^{N-1}\left(\delta_{y} u_{m, 0}^{k}\right)^{4}+\sum_{n=1}^{N-1}\left(\delta_{x} u_{0, n}^{k}\right)^{4}\right],
\end{aligned}
$$


which also provides a consistent approximation of the equation of total energy (2.5). Furthermore, it is easy to check that the discrete rate of change of energy provided by our method is given by the equation

$$
\begin{aligned}
\frac{\delta_{t} E^{k}}{\Delta t}= & -\gamma \sum_{m=1}^{N-1} \sum_{n=1}^{N-1}\left(\frac{\delta_{t} u_{m, n}^{k}}{\Delta t}\right)\left(\frac{\delta_{t}^{1} u_{m, n}^{k}}{2 \Delta t}\right)-c^{2} \sum_{m=1}^{N-1}\left(\frac{\delta_{y} u_{m, 0}^{k+1}+\delta_{y} u_{m, 0}^{k}}{2}\right) \frac{\delta_{t} u_{m, 0}^{k}}{\Delta t} \\
& -c^{2} \sum_{n=1}^{N-1}\left(\frac{\delta_{x} u_{0, n}^{k+1}+\delta_{x} u_{0, n}^{k}}{2}\right) \frac{\delta_{t} u_{0, n}^{k}}{\Delta t}-\beta \sum_{m=1}^{N-1} \frac{\sigma_{y} u_{m, 0}^{k}}{4} \frac{\delta_{t} u_{m, 0}^{k}}{\Delta t} \\
& -\beta \sum_{n=1}^{N-1} \frac{\sigma_{x} u_{0, n}^{k}}{4} \frac{\delta_{t} u_{0, n}^{k}}{\Delta t},
\end{aligned}
$$

which is a consistent approximation of the derivative of the total energy of the system (2.1) with respect to time.

\section{Simulations}

Let us consider a lattice consisting of $N \times N$ sites with $N=25$. We fix a constant damping coefficient equal to 0.01 , a coefficient of nonlinearity of 1 , a coupling factor equal to 5 , and a driving frequency in the forbidden band gap equal to 2.5. Computationally, we fixed a time step of 0.05 , and a time period of length 1000 .

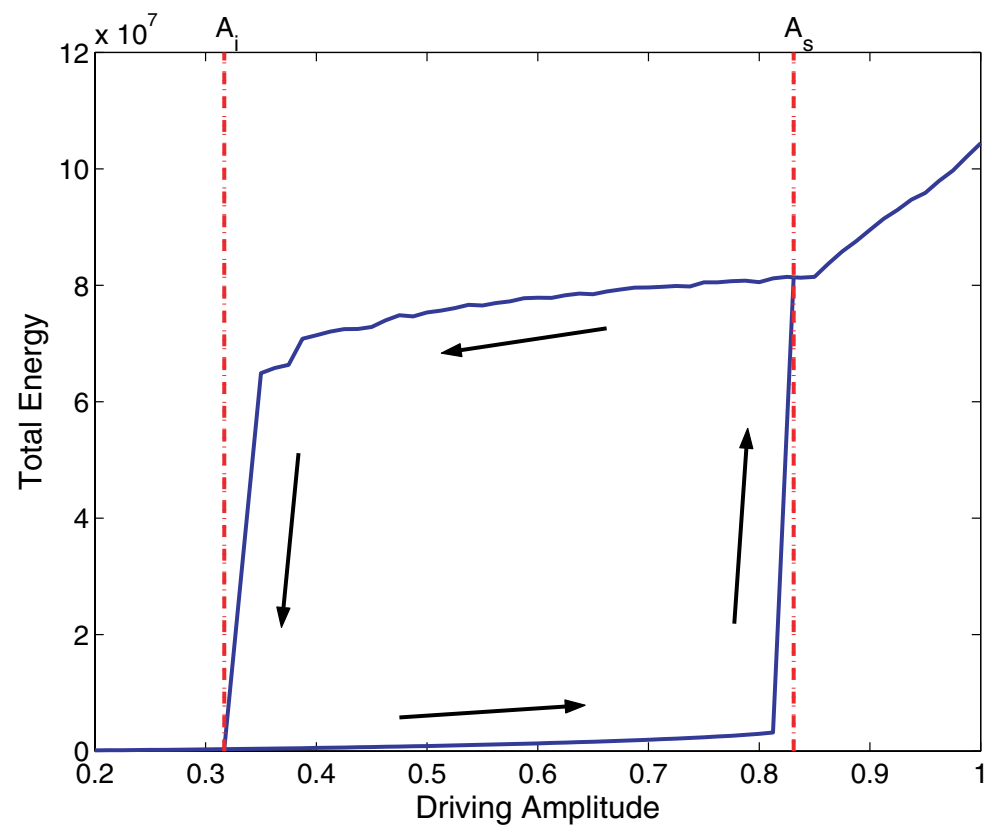

Fig. 1. Graph of total energy of a system governed by (2.1) versus driving amplitude, initially at rest with each site in resting position, with parameters $T=1000, N=25, c=5, \beta=1, \gamma=0.01, \Omega=2.5$, and $\Delta t=0.05$. The system was harmonically perturbed in two boundaries by (2.2), and void Neumann conditions were imposed on the other two. The vertical dashed lines represent the critical thresholds of infratransmission and supratransmission ( $A_{i}$ and $A_{s}$, respectively), and the arrows represent the direction of increase or decrease of the driving amplitude. 


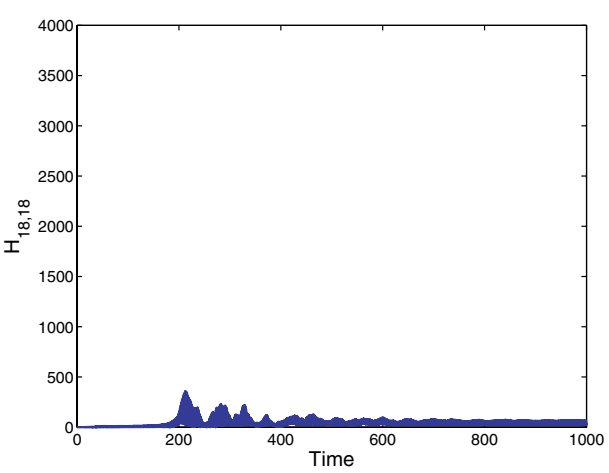

(a) $A=0.835$

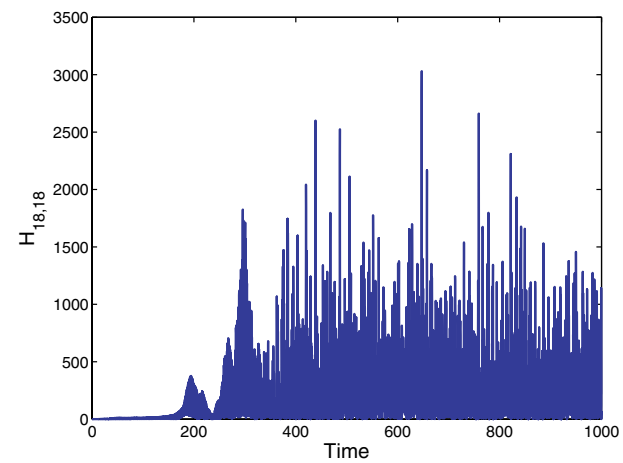

(c) $A=0.435$

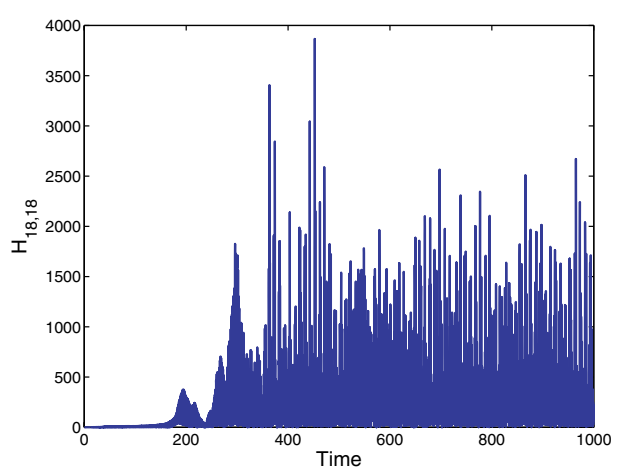

(b) $A=0.845$

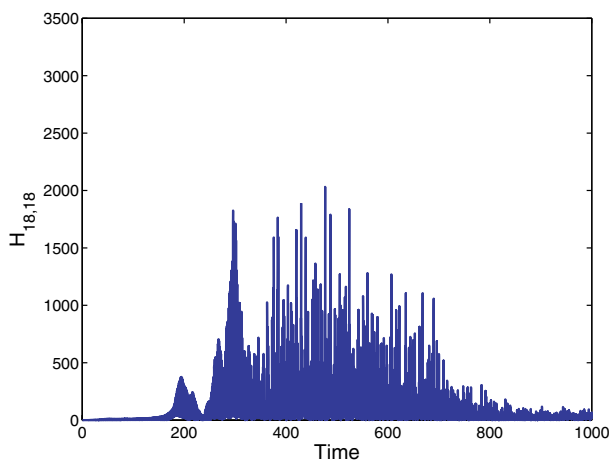

(d) $A=0.425$

Fig. 2. Graphs of local energy versus time of the node located in site $(18,18)$, of a system governed by $(2.1)$ with $N=25$. The following parametric and computational values were employed in the simulations: $\Omega=2.5$, $\gamma=0.01, \beta=1, c=5, T=1000$, and $\Delta t=0.05$. In the top row, the driving amplitude is increased in finite time from a value of zero to the value $A$ given in each case. Meanwhile, in the bottom row, a system which has already reached supratransmission is considered, and the value of the driving amplitude is decreased from the supratransmission threshold 0.845 to the actual given value $A$.

The boundaries of the system were perturbed using the sinusoidal function

$$
\varphi(t)=A(t) \sin (\Omega t),
$$

where the nonnegative function $A(t)$ modulates the amplitude, increasing it progressively from 0 to a fixed constant $A^{\prime}$, in order to avoid the possible generation of shock waves. In our case, $A(t)$ was a linear function, and the value $A^{\prime}$ was reached after 50 time units. We employed our method for increasing values of $A^{\prime}$, and we kept track of the total energy of the system until a sudden and massive peak of energy was registered, reaching thus the supratransmission threshold $\left(A_{s}\right)$. Next, we carried out simulations but now considering a system which was already transmitting energy from the driving boundary. Clearly, the values given to $A^{\prime}$ on the second set of simulations decreased from the supratransmission threshold, and so $A(t)$ likewise decreased from $A_{s}$ down to $A^{\prime}$ for the first 50 units of time. A significant drop in the total energy marked the presence of the infratransmission threshold $\left(A_{i}\right)$. In fact Fig. 1 presents the results of our simulations, together with the directions of increase or decrease of the driving amplitude. 
According to our simulations, we have found that $A_{s} \approx 0.835$ and $A_{i} \approx 0.310$. We wish to approximate these values more accurately by studying the dynamics of a specific node of the lattice; particularly, we fixed the node located in the site $(18,18)$, and took two values for the driving amplitudes: one right before supratransmission starts, and the other right after the supratransmission threshold. In similar fashion, we proceeded systematically to determine the infratransmission threshold to an accuracy of within 0.01. The first and second rows of Fig. 2 show that the actual values for $A_{s}$ and $A_{i}$ are 0.845 and 0.415 , respectively.

\section{Application}

In the present section, we employ our model to send binary signals taking advantage of the simplicity to pass from a state of supratransmission to one of infratransmission and vice versa. This was done by using a continuous function to regulate the amplitude according to the corresponding bit that we intended to send. We took $A_{s}=0.85$ and $A_{i}=0.13$ in order to facilitate the transition from one state to another.

Let $S=b_{1} b_{2} \cdots b_{n}$ be the chain consisting of binary bits to be transmitted into the system. We employed the following function to control the amplitude

$$
\begin{aligned}
& A\left(t_{k}, b_{i}\right)= \begin{cases}0.36 \sin \left[\frac{\pi}{100}(t+150)\right]+0.49, & b_{i}=1, \\
0.1, & b_{i}=0,\end{cases} \\
& P_{k-1} \leq t_{k} \leq P_{k}
\end{aligned}
$$

where $k$ is a positive integer and $P_{k}=200 k$, that is, the period of the function when $b_{i}=1$ multiplied by $k$. We sent a 10-bit message given by $S=1101010011$ using the amplitude

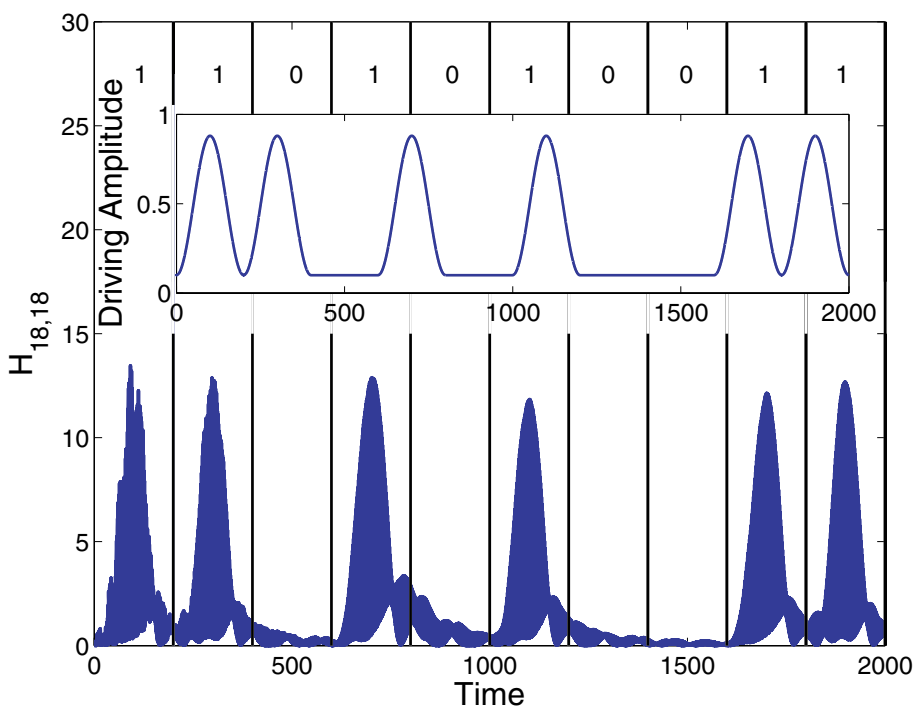

Fig. 3. Graphs of local energy versus time of the node located in site $(18,18)$, of a system governed by $(2.1)$ with $N=25$. The following parametric and computational values were employed in the simulations: $\Omega=2.5$, $\gamma=0.01, \beta=1, c=5, T=1000$, and $\Delta t=0.05$. The graph is the result of transmitting the binary code "1101010011" when perturbing the boundary by the amplitude modulation function presented in the inset. 


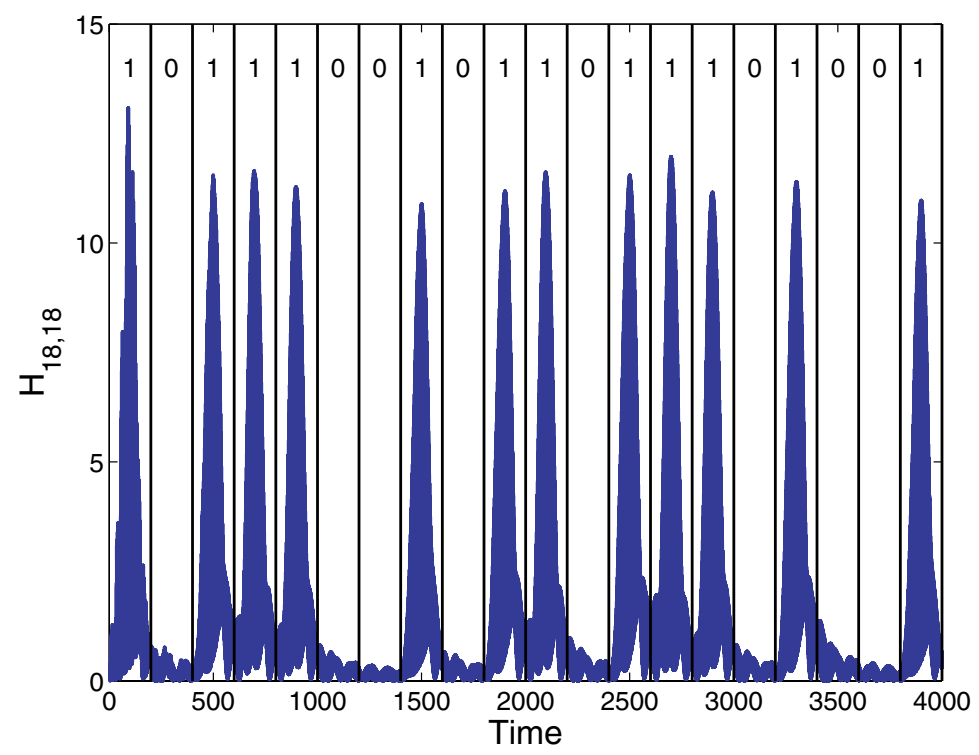

Fig. 4. Graphs of local energy versus time of the node located in site $(18,18)$, of a system governed by (2.1) with $N=25$. The following parametric and computational values were employed in the simulations: $\Omega=2.5, \gamma=0.01, \beta=1, c=5, T=1000$, and $\Delta t=0.05$. The graph is the result of transmitting the binary code "10111001011011101001" when perturbing the boundary.

function described above. The results are shown in Fig. 3 as a graph of the local energy at site $(18,18)$ versus time, and they evidence the fact the binary information is transmitted accurately from the driving boundary.

Having seen that the information was transmitted without any difficulties we send a message twice as long as the first, in order to verify that the reliability of the process is independent of the length of the signal. We proceeded to propagate the binary message "10111001011011101001", and Fig. 4 summarizes the result of our simulation, confirming that no distortion of information was generated during the process.

\section{Conclusion}

A $\beta$-Fermi-Pasta-Ulam lattice in two space dimensions can effectively be used to send binary data by modulating the driving amplitude of two adjacent boundaries between the supra- and infratransmission thresholds; a sinusoidal function fulfills this task in a very efficient way. This method for transmitting binary signals is reliable and "economical" in view of the fact that the system never completely ceases to irradiate energy; it simply stays idle until a proper variation of the driving amplitude changes it between conducting and insulating regimes.

\section{Acknowledgments}

The authors wish to thank the anonymous referee for her/his thorough review of the manuscript and for her/his invaluable comments. One of us (JEMD) wishes to acknowledge support from Dr. Álvarez Rodríguez, dean of the Faculty of Sciences of the Universidad Autónoma de Aguascalientes, and from Dr. Avelar González, head of the Office for 
Research and Graduate Studies of the same university, in the form of computational resources. The present work represents a set of partial results under project PIM08-1 at this institution.

\section{References}

[1] A. Ponno and D. Bambusi, Korteweg-de Vries equation and energy sharing in Fermi-PastaUlam, Chaos: Int. J. Nonlin. Sci. 15 (2005) 015107.1-015107.5.

[2] A. R. Janzen and J. W. Leech, Lattice and molecular dynamics, Can. J. Chem. 66 (1988) 852-856.

[3] B. Rink, Direction-reversing traveling waves in the even Fermi-Pasta-Ulam lattice, J. Nonlin. Sci. 12 (2008) 479-504.

[4] D. Blackmore, R. Samulyak and A. Rosato, New mathematical models for particle flow dynamics, J. Non-Linear Math. Phys. 6 (1999) 198-221.

[5] D. Chevrieux, R. Khomeriki and J. Leon, Theory of a Josephson junction parallel array detector sensitive to very weak signals, Phys. Rev. B 73 (2006) 214516.

[6] D. L. Shepelyansky, Low-energy chaos in the Fermi-Pasta-Ulam problem, Nonlin. 10 (1997) 1331-1338.

[7] E. Fermi, J. Pasta and S. Ulam, Studies of nonzlinear problems, Los Alamos Report LA-1490 (1955).

[8] P. G. Estévez, E. Conde and P. R. Gordoa, Unified approach to Miura, Bäcklund and Darboux transformations for nonlinear partial differential equations, J. Non-Linear Math. Phys. 5 (1998) $82-114$.

[9] F. Geniet and J. Leon, Energy transmission in the forbidden band gap of a nonlinear chain, Phys. Rev. Lett. 89 (2002) 134102.

[10] F. Geniet and J. Leon, Nonlinear supratransmission, J. Phys.: Condens. Matter 15 (2003) 2933-2949.

[11] G. Zhang and B. Li, Thermal conductivity of nanotubes revisited: Effect of chirality, isotope impurity, tube length, and temperature, J. Chem. Phys. 123 (2005) 114714.

[12] H. Kantz, R. Livi and S. Ruffo, Equipartition thresholds in chains of anharmonic oscillators, J. Stat. Phys. 76 (1994) 627-643.

[13] I. A. Butt and J. A. D. Wattis, Discrete breathers in a two-dimensional Fermi-Pasta-Ulam lattice, J. Phys. A: Math. Gen. 39 (2006) 4955-4984.

[14] I. A. Butt and J. A. D. Wattis, Discrete breathers in a two-dimensional hexagonal Fermi-Pasta-Ulam lattice, J. Phys. A: Math. Theor. 40 (2007) 1239-1264.

[15] J. E. Macías-Díaz, A numerical method with properties of consistency in the energy domain for a class of dissipative nonlinear wave equations with applications to a Dirichlet boundary-value problem, Z. Angew. Math. Mech. 88 (2008) 828-846.

[16] J. E. Macías-Díaz, Bistability of a two-dimensional Klein-Gordon system as a reliable means to transmit monochromatic waves: A numerical approach, Phys. Rev. E $\mathbf{7 8}$ (2008) 056603.

[17] J. E. Macías-Díaz, Numerical study of the transmission of energy in discrete arrays of sineGordon equations in two space dimensions, Phys. Rev. E 77 (2008) 016602.

[18] J. E. Macías-Díaz and A. Puri, An application of nonlinear supratransmission to the propagation of binary signals in weakly damped, mechanical systems of coupled oscillators, Phys. Lett. A 366 (2007) 447-450.

[19] J. E. Macías-Díaz and A. Puri, On the propagation of binary signals in damped mechanical systems of oscillators, Physica D 228 (2007) 112-121.

[20] J. E. Macías-Díaz and A. Puri, On the transmission of binary bits in discrete Josephson-junction arrays, Phys. Lett. A 372 (2008) 5004-5010.

[21] J.-G. Caputo, J. Leon and A. Spire, Nonlinear energy transmission in the gap, Phys. Lett. A 283 (2001) 129-135. 
[22] J. Leon, Nonlinear supratransmission as a fundamental instability, Phys. Lett. A 319 (2003) $130-136$.

[23] J. Leon and A. Spire, Gap soliton formation by nonlinear supratransmission in Bragg media, Phys. Lett. A 327 (2004) 474-480.

[24] M. Kastner, Energy threshold for discrete breathers, Phys. Rev. Lett. 92 (2004) 104301.

[25] M. Peyrard, S. C. López and D. Angelov, Fluctuations in the DNA double helix, Euro. Phys. J. 147 (2007) 173-189.

[26] R. Khomeriki and J. Leon, Bistability in sine-Gordon: The ideal switch, Phys. Rev. E 71 (2005) 056620 .

[27] R. Khomeriki, S. Lepri and S. Ruffo, Nonlinear supratransmission and bistability in the Fermi-Pasta-Ulam model, Phys. Rev. E 70 (2004) 066626.

[28] S. Flach and A. Gorbach, Discrete breathers in Fermi-Pasta-Ulam lattices, Chaos: Int. J. Nonlin. Sci. 15 (2005) 015112.1-015112.10.

[29] S. Flach, K. Kladko and S. Takeno, Acoustic breathers in two-dimensional lattices, Phys. Rev. Lett. 79 (1997) 4838-4841.

[30] T. Mai, A. Dhar and O. Narayan, Equilibration and universal heat conduction in Fermi-PastaUlam chains, Phys. Rev. Lett. 98 (2007) 184301.

[31] Y. Wakabayashi, M. Tokeshi, A. Hibara, D.-L. Jiang, T. Aida and T. Kitamori, Morphological dependence of radiative and nonradiative relaxation energy balance in photoexcited aryl ether dendrimers as observed by fluorescent and thermal lens spectroscopies, J. Phys. Chem. B 105 (2001) 4441-4445. 